\title{
Tumor Boards: Beyond the Patient Care Conference
}

\author{
Abdul Rahman Jazieh
}

Published online: 5 March 2011

(C) is Springer 2011

\section{Introduction}

Tumor Boards (TB) are essential components of contemporary cancer care $[1,2]$. They provide a multidisciplinary expert approach to patients in a setting where all pertinent data can be reviewed and assessed to synthesize the best treatment plan for a particular patient.

However, tumor boards have multiple other benefits and functions that go beyond just formulating care plans for cases discussed. Benefits of tumor boards include quality improvement, program development, and professional career enhancement.

On the other hand, there are multiple challenges related to the formation, function, and maintenance of TBs. Overcoming these challenges can be facilitated by a better understanding of the multifaceted functions of tumor boards; and the tremendous value added by these tumor boards not only to patient care, but also to the health care professionals and the organization at large.

In this manuscript, the functions of tumor boards will be discussed presenting ideas that can potentially be utilized to improve the work of oncology multidisciplinary teams.

\section{Establishing Tumor Boards}

In order to establish a successful tumor board, certain issues should be addressed in a systematic way including membership, leadership, support staff, infrastructure, frequency of meetings, case loads, areas of interest, and the process of conducting the tumor board.

A. R. Jazieh $(\bowtie)$

Department of Oncology (MC 1777), King Abdulaziz Medical

City, King Saud bin Abdulaziz University for Health Sciences, P.O. Box 22490, Riyadh 11426, Kingdom of Saudi Arabia

e-mail: jazieha@ngha.med.sa
Tumor Board Membership

Tumor boards should include specialists and subspecialists of medical and surgical fields such as medical oncologists, surgical oncologists, radiation oncologists in addition to radiologists, pathologists, and other support professional and administrative staff.

It is very important to have more than one physician in each major specialty to enrich the intellectual reservoir of the TB and to assure the continuity of TB, and to improve patient care. Encouraging junior physicians, trainees, and students to attend is important to improve their training experience and instill the culture of team work.

\section{Tumor Board Leadership}

Each TB should have a facilitator to oversee the various activities of the board. The facilitator is the orchestrator of the team work to come up with the best performance. The facilitator will monitor attendance of various specialists, encourage participation, assure consensus building for final recommendations and facilitate communication among team members. Therefore, the interpersonal skills of the facilitator are crucial for the success of the TB [3].

The facilitator specialty can be from any discipline pertinent to the specific area of the TB. However, rotating the leadership of the tumor board at reasonable intervals should be considered to encourage engagement of different members and disciplines in the process.

\section{Support Staff}

Having support staff to maintain the basic functions of the TBs is crucial including secretarial and tumor registry staff 
support. Participation of other staff may be required depending on the complexity of the TB and its functions.

\section{Infrastructure}

Availability of a conference room that is accessible to members is crucial. Electronic access to records, imaging and pathology will certainly enhance the function of the team.

Telemedicine capacity may facilitate attendance of remote individuals as experts or as members present their cases or for educational purposes [4].

\section{Areas of Interest}

In facilities with limited cancer cases and small oncology services, one general tumor board may suffice. However, for specialized cancer hospitals or hospitals with large cancer programs, disease or site specific TBs are encouraged and may be needed to handle the volume and the complexity of the cases.

\section{The Process of Tumor Boards}

\section{Before the Meeting}

Deadline for case submission should be set in advance to allow adequate preparation and review of slides and imaging films. Pertinent information in the form of case summary should be sent in advance so members understand the purpose of the presentation.

\section{During the Meeting}

Balanced discussion should be conducted with a focus on evidence-based opinions and adherence to guidelines, as much as possible.

Dissenting opinions should be respected and the discussion should not turn into personal argument. At the conclusion of the meeting, a consensus recommendation should be reached. A follow-up discussion of certain cases should be scheduled.

\section{After the Meeting}

Documentation of the TB recommendations should be made available in patient's records.

Follow-up on the recommendations of the TB should be carried out by the primary physician caring for the patient.

Follow-up presentation to TB should be arranged, if necessary. This will be important to discuss further management and to have feedback on the outcome of the board recommendation, which will have a tremendous educational value.

\section{Frequency of the Meeting}

The TB meeting schedule depends on many factors including the number of cases and the availability of members. Therefore, meeting frequency may range from more than once a week to once a month. The most important factor is the timeliness of the meeting for patients care as new and complex cases should be addressed, one way or another, in a multidisciplinary fashion. If there are cases that have pressing need to be discussed sooner than the next scheduled meeting, then an ad hoc meeting of the whole tumor board or pertinent members can be arranged to discuss the particular case.

\section{What are the Various Potential Functions of TB?}

The following are the potential activities that TB members can conduct to reap the most benefits of the team:

1. Multidisciplinary Care Conference: This is the basic and classical function of TB. The TB offers a platform for multiple expert consultations in a timely fashion resulting in practical and relevant recommendation to manage the patient. It is known fact that TB may impact the management of the patients discussed [510].

2. Quality Improvement (QI): $\mathrm{TB}$ can be a major catalyst for quality improvement process in the institution. It acts like a peer review of the care and critical appraisal of the system [11]. Each TB may develop a QI project pertinent to the area such as improving time to diagnosis, or shortening time from diagnosis to definitive treatment, etc.

Furthermore, members may develop QI process related to their discipline such as investigating discrepancy in pathology or imaging reports and impact on treatment decisions.

The TB may improve patient care by developing clinical guidelines and pathways for patient care and monitor adherence to these guidelines.

3. Improving Clinical Services: TB can be the foundation for an actual multidisciplinary clinic where cases are not just reviewed or discussed, but actually the patient is seen and examined by one or more of the team members which assures timely decision and execution of that decision.

In certain setting and with the availability of expertise and infrastructure, a center of excellence or expertise (COE) may be created to provide more sophisticated services and highly specialized care to the patients. The COE projects better visibility and prominence of the team and the institution. Therefore, the multidisciplinary team function can be categorized 
into four levels as illustrated in the pyramid of multidisciplinary team function (Fig. 1).

a. Basic Tumor Boards: provide the basic cancer care conference. It is essential to establish the cancer care conference as a foundation to achieve more complex activities and projects.

b. Enhanced Tumor Board: which have additional components such as research, or educational/training or quality improvement activities.

c. Multidisciplinary Clinic: provide clinical care directly to patients [10].

d. Center of Excellence: provide highly specialized, sophisticated, and unique services to patients.

4. Education: TB is an effective educational venue with practical teaching on real cases. The educational value can be enhanced by adding short didactic presentations about topic of interest related to case discussed [5, 12, 13].

Furthermore, TB may lead the institutional public and professional educational activities. Activities may include symposia or workshops in the area of interest organized by the team members.

5. Cancer Research: As cancer care is multidisciplinary, successful cancer research is also dependent on team approach. The team can brain storm about research ideas, discuss feasibility of a particular study, and help recruit patients to clinical trials.

6. Professional Development: TB offers junior physicians and staff practical mentoring with access to multiple clinical expertise, research, and educational opportunities.

7. Accreditations: Cancer center accreditation by American College of Surgeons and others is dependent, in part, on multidisciplinary team work and tumor board performance [14].
How to Improve Participation of TB Members?

Motivation and commitment of TB members are essential for its success. The following tips and ideas may improve the members' involvement in the TB:

1. Revisit the issue of timing of the TB if attendance declines.

2. Ensure participation of more than one member from each discipline, if feasible.

3. Involve the departments leaders in the process.

4. Generate and track attendance reports for feedback.

5. Distribute tasks to different members to encourage involvement and buy in such as research, QI, education, etc.

6. Rotate leadership of the TB.

7. Use telemedicine to access international expertise to enrich the team experience [4].

8. Make the TB approval as a prerequisite for certain therapeutic procedures such as operating room scheduling, concurrent chemo radiotherapy, etc.

9. Integrate $\mathrm{TB}$ utilization in the institutional peer review process and physician performance evaluation such as morbidity and mortality review, medical practice review etc. Cancer cases discussed by these committees should be reviewed for tumor board presentation and if not done, reasons should be investigated.

In summary, multidisciplinary cancer care can start as a cancer care conference known as "tumor board" but it can evolve based on the individual team resources and performance into higher forms of team work benefiting patients, professionals, and health care organizations.
Fig. 1 The pyramid of multidisciplinary functions

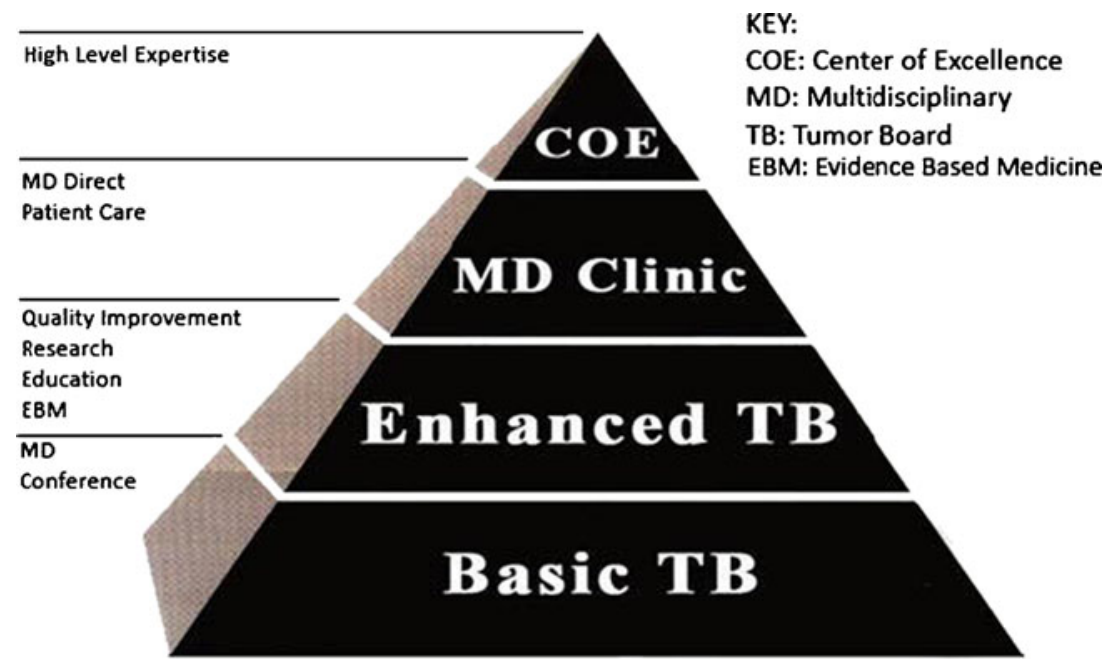




\section{References}

1. Westin T, Stalfors J (2008) Tumour boards/multidisciplinary head and neck cancer meetings: are they of value to patients, treating staff or a political additional drain on health care resources. Curr Opin Otolaryngol Head Neck Surg 16:103-107

2. Rabinowitz B (2004) Interdisciplinary breast cancer care: declaring and improving the standard. Oncology (Williston Park) 18:1263-1268, discussion 1268-70, 1275

3. Bakemeier RF, Beck S, Murphy JR (1995) Educational and consultative functions, topics, and methods of hospital general tumor conferences. J Cancer Educ 9:217-225

4. Hazin R, Qaddoumi I (2010) Teleoncology: current and future applications for improving cancer care globally. Lancet Oncol 11:204-210

5. Gatcliffe TA, Coleman RL (2008) Tumor board: more than treatment planning - a 1-year prospective survey. J Cancer Educ 23:235-237

6. Petty K, Vetto JT (2002) Beyond doughnuts: tumor board recommendations influence patient care. J Cancer Educ 17:97100
7. Chang JH, Vines E, Bertsch H et al (2001) The impact of a multidisciplinary breast cancer center on recommendations for patient management. Cancer 91:1231-2351

8. Forrest LM, McMillan DC, McArdle CS et al (2005) An evaluation of the impact of a multidisciplinary team, in a single centre, on treatment and survival in patients with inoperable nonsmall-cell lung cancer. Br J Cancer 93:977-978

9. Newman EA, Guest AB, Helvie MA et al (2006) Changes in surgical management resulting from case review at a breast cancer multidisciplinary tumor board. Cancer 107:2346-2351

10. Pawlik TM, Laheru D, Hruban RH et al (2008) Evaluating the impact of a single-day multidisciplinary clinic on the management of pancreatic cancer. Ann Surg Oncol 15:2081-2088

11. Gross E (1987) The role of the tumor board in a community hospital. CA Cancer J Clin 37:88-92

12. Radecki SE, Nyquist JG, Gates JD et al (1995) Educational characteristics of tumor conferences in teaching and non-teaching hospitals. J Cancer Educ 9:204-216

13. Strobel SL (2006) Enhancing the pathologist's role at hospital tumor boards. Ann Clin Lab Sci 36:243-247

14. Santoso JT, Schwertner B, Coleman RL et al (2004) Tumor board in gynecologic oncology. Int J Gynecol Cancer 14(2):206-209 